A $\underset{\text { publications }}{\mathrm{C}}$ Rec. Nat. Prod. 13:4 (2019) 296-300

records of natural products

\title{
A New Pericarbonyl Lignan from Amauroderma rude
}

\section{Miao Dong ${ }^{1}$, Zuhong $\mathrm{Ma} \odot^{2}$, Qiaofen Yang $\odot^{2}$, Qiuyue $\mathrm{Hu} \odot^{2}$, Yanqing $\mathrm{Ye} \odot^{2, *}$ and Min Zhou ${ }^{1, *}$}

\author{
${ }^{1}$ Key Laboratory of Chemistry in Ethnic Medicinal Resources, State Ethnic Affairs Commission \& \\ Ministry of Education, Yunnan Minzu University, Kunming 650031, P.R. China \\ ${ }^{2}$ School of Chemistry and Environment, Yunnan Minzu University, Kunming 650031, P.R. China
}

(Received October 24, 2018; Revised November 29, 2018; Accepted November 30, 2018)

\begin{abstract}
A new pericarbonyl lignan (1), named amaurolignan A was isolated from an ethanol extract of the fruiting bodies in Amauroderma rude of family Ganodermataceae, together with two known lignans, 4-methoxymatairesinol $4{ }^{\prime}-\beta$-D-glucoside (2) and lappaol F (3). The structures of compounds (1-3) were elucidated using NMR and MS spectroscopic methods.
\end{abstract}

Keywords: Pericarbonyl lignan; amaurolignan A; Amauroderma rude. (C) 2019 ACG Publications. All rights reserved.

\section{Introduction}

"Lingzhi" is a mushroom that has been renowned in China for more than 2000 years because of its claimed medicinal properties and symbolic fortune, which translates as 'Ganodermataceae' in a broad sense, and in a narrow sense it represents the highly prized medicinal Ganoderma species distributed in East Asia [1]. Its medicinal properties include anti-aging, lowering blood pressure, improving immunity, and preventing and treating various cancers, chronic bronchitis, gastric ulcers, hepatitis, neurasthenia and thrombosis [2-4]. The medicinal effects of many mushrooms such as Ganoderma lucidum, Lentinula edodes, Agaricus blazei, Antrodia camphorate and Grifola frondosaI come from their metabolites including polysaccharides, triterpenes, lucidenic acids, adenosine, ergosterol, glucosamine and cerebrosides [5-8]. This caused us to look further into another mushroom, Amauroderma rude (Berk.) Torrend, which called 'Xuezhi' in China and some species in this genus have been newly recognized as medicinal fungus [9-10]. The genus Amauroderma contains approximately 30 species and most of the species are widespread in tropical areas and rarely studied [11].

In an attempt to look for potential active substances from the Amauroderma rude, the isolation and chemical identification of one new pericarbonyl lignan (1) and two known lignans, 4-methoxymatairesinol 4'- $\beta$-D-glucoside (2) [12] and lappaol F (3) [13] (Figure 1) from the ethanol extract of A. rude were taken. Details of the isolation and identification of compound $\mathbf{1}$ are presented herein and the known compounds 2-3 were compared of spectroscopic data with those reported.

\footnotetext{
* Corresponding author: E-Mail: zhouminynun@163.com 


\section{Materials and Methods}

\subsection{Instrumentation and Reagents}

The UV data was detected by Shimadzu UV-2401A. Mass spectra were performed on a VG Autospec-3000 spectrometer under $70 \mathrm{eV}$. 1D and 2D NMR spectra were recorded on Bruker AV-400 spectrometer with TMS as the internal standard (Bruker BioSpin Group). $\mathrm{SiO}_{2}$ (100-200 mesh, Qingdao Marine Chemical Inc., China), Lichroprep RP-18 gel (40-63 $\mu \mathrm{m}$, Merck, Darmstadt, Germany), and MCI gel (75-150 $\mu \mathrm{m}$, Mitsubishi Chemical Corporation, Tokyo, Japan) were used for column chromatography. Semi-preparative HPLC was performed on an Agilent 1100 liquid chromatography with a Welch Ultimate XB-Phenyl or Ultimate XB-C18 $(10 \mu \mathrm{m}, 4.6 \mathrm{~mm} \times 25 \mathrm{~cm})$. Preparative HPLC was performed on a Shimadzu LC-8A preparative liquid chromatograph with a Shimadzu PRC-ODS (K) column. Fractions were monitored by TLC and spots were visualized by heating silica gel plates sprayed with $8 \% \mathrm{H}_{2} \mathrm{SO}_{4}$ in $\mathrm{EtOH}$. All solvents including petroleum ether $\left(60-90^{\circ} \mathrm{C}\right)$ were distilled prior to use.

\subsection{Plant Materials}

The fruiting bodies of Amauroderma rude were collected in January 2017 from Ruili State, Yunnan Province, China, and were authenticated by Prof. Min Zhou (Key Laboratory of Chemistry in Ethnic Medicinal Resources, Yunnan Minzu University). A sample (201701A) was preserved in Yunnan Minzu University, Kunming.

\subsection{Extraction}

The chopped, dried fruiting bodies of $A$. rude $(40 \mathrm{~kg}$ ) was extracted with $95 \%$ ethanol solution heated under reflux ( 2 times $/ 60 \mathrm{~min}$ ), at $20^{\circ} \mathrm{C}$ to give a residue $(3 \mathrm{~kg}$ ). The extract was suspended in pure water and partitioned with EtOAc. The EtOAc fraction $(1.3 \mathrm{~kg})$ was partitioned with a silica gel column (dichloromethane: methanol, from 1:0 80:1 20:1 8:1 5:1 2:1, each $5 \mathrm{~L}$ ) to afford six subfractions (Fr. AF). Fr. D $(110 \mathrm{~g})$ was further eluted with MCI column using a stepwise gradient of $\mathrm{MeOH} / \mathrm{H}_{2} \mathrm{O}$ (from $30 \% \sim 55 \% \sim 90 \% \sim 100 \%$, each $4 \mathrm{~L}$ ) to afford three fractions (Fr. D1-D3). Fr. D2 (45.0 g) was loaded onto ODS (MeOH/ $\mathrm{H}_{2} \mathrm{O} 40 \% \sim 60 \% \sim 80 \% \sim 100 \%$, each $2 \mathrm{~L}$ ) to give four fractions (Fr. D2-1 4). Fr. D2-2 (15.5 g) was separated over YMC-Pack ODS-A $(20 \times 250 \mathrm{mml}$. D.S, $5 \mu \mathrm{m})$ prep. HPLC $\left(68 \% \mathrm{MeOH} / \mathrm{H}_{2} \mathrm{O}\right)$, yielding $\mathbf{2}(25.0 \mathrm{mg})$ and $\mathbf{3}(30.6 \mathrm{mg})$. Fr. D2-3 (10.0 g) was further eluted with a silica gel (dichloromethane/acetone, from 80:1 20:1 10:1 8:1 2:1, each $2 \mathrm{~L}$ ) and separated over semi-prep. HPLC $\left(50 \% \mathrm{MeOH}-\mathrm{H}_{2} \mathrm{O}\right)$ to yield $1(22.0 \mathrm{mg})$.

Amaurolignan A (1): yellow oil; UV $\lambda_{\max } 230,280$ and $330 \mathrm{~nm} ;{ }^{1} \mathrm{H}$ NMR $\left(\mathrm{CD}_{3} \mathrm{OD}\right)$ and ${ }^{13} \mathrm{C} \mathrm{NMR}\left(\mathrm{CD}_{3} \mathrm{OD}\right)$ see Table 1; HR-ESIMS $m / z 329.0669$ [M-H] ${ }^{-}$(calculated for $\mathrm{C}_{17} \mathrm{H}_{14} \mathrm{O}_{7}, 329.0667$ ).

4-Methoxymatairesinol 4'- $\beta$-D-Glucoside (2): White oily liquid; ${ }^{1} \mathrm{H}$ NMR $\left(400 \mathrm{MHz}, \mathrm{CD}_{3} \mathrm{OD}\right): \delta_{\mathrm{H}} 7.07$ $\left(1 \mathrm{H}, \mathrm{d}, J=8.2 \mathrm{~Hz}, \mathrm{H}-5^{\prime}\right), 6.83(1 \mathrm{H}, \mathrm{d}, J=8.6 \mathrm{~Hz}, \mathrm{H}-5), 6.76(1 \mathrm{H}, \mathrm{s}, \mathrm{H}-2), 6.66(1 \mathrm{H}, \mathrm{d}, J=8.2 \mathrm{~Hz}, \mathrm{H}-6)$, $6.61\left(2 \mathrm{H}, \mathrm{d}, J=5.9 \mathrm{~Hz}, \mathrm{H}-2^{\prime} / 6^{\prime}\right), 4.19\left(1 \mathrm{H}, \mathrm{t}, J=8.2 \mathrm{~Hz}, \mathrm{H}-9^{\prime} \mathrm{a}\right), 3.94\left(1 \mathrm{H}, \mathrm{t}, J=8.2 \mathrm{~Hz}, \mathrm{H}-9^{\prime} \mathrm{b}\right), 3.87(1 \mathrm{H}$, d, $J=12.0 \mathrm{~Hz}, \mathrm{H}-\mathrm{Glc}-6 \mathrm{a}), 3.80\left(1 \mathrm{H}, \mathrm{s}, \mathrm{H}-\mathrm{OCH}_{3}-4\right), 3.76\left(6 \mathrm{H}, \mathrm{s}, \mathrm{H}-3 / 3^{\prime}\right), 3.70(1 \mathrm{H}, \mathrm{d}, J=11.3 \mathrm{~Hz}, \mathrm{H}-\mathrm{Glc}-$ 6b), $3.50(2 \mathrm{H}, \mathrm{d}, J=6.7 \mathrm{~Hz}, \mathrm{H}-\mathrm{Glc}-2,3), 3.42(2 \mathrm{H}, \mathrm{s}, \mathrm{H}-\mathrm{Glc}-4,5), 2.92(1 \mathrm{H}, \mathrm{dd}, J=13.9,5.3 \mathrm{~Hz}, \mathrm{H}-7 \mathrm{a})$, $2.82(1 \mathrm{H}, \mathrm{dd}, J=13.9,7.2 \mathrm{~Hz}, \mathrm{H}-7 \mathrm{~b}), 2.68(1 \mathrm{H}, \mathrm{dd}, J=13.2,7.2 \mathrm{~Hz}, \mathrm{H}-8), 2.56(2 \mathrm{H}, \mathrm{d}, J=6.3 \mathrm{~Hz}, \mathrm{H}-$ $\left.7 \mathrm{a}^{\prime} / 7 \mathrm{~b}^{\prime}\right), 2.49\left(1 \mathrm{H}, \mathrm{dd}, J=14.6,7.5 \mathrm{~Hz}, \mathrm{H}-8^{\prime}\right) .{ }^{13} \mathrm{C}$ NMR (400 MHz, CD $\left.{ }_{3} \mathrm{OD}\right): \delta_{\mathrm{C}} 132.7(\mathrm{C}-1), 114.8(\mathrm{C}-2)$, 149.1 (C-3), 150.4 (C-4), 113.1 (C-5), 123.0 (C-6), 35.4 (C-7), 47.6 (C-8), 134.2 (C-1'), 113.6 (C-2'), 150.6 (C-3'), 146.8 (C-4'), 117.8 (C-5'), 122.1 (C-6'), 38.9 (C-7'), 42.5 (C-8'), 72.9 (C-9'), 102.9 (Glc-1), 74.9 (Glc-2), 77.8 (Glc-3), 71.3 (Glc-4), 78.1 (Glc-5), 62.5 (Glc-6), $56.7\left(\mathrm{OCH}_{3}-4\right), 56.5\left(\mathrm{OCH}_{3}-3 / 3^{\prime}\right)$.

Lappaol F (3): ${ }^{1} \mathrm{H}$ NMR (400 MHz, $\left.\mathrm{CD}_{3} \mathrm{OD}\right): \delta_{\mathrm{H}} 6.63(1 \mathrm{H}, \mathrm{br} \mathrm{s}, \mathrm{H}-2), 6.57(2 \mathrm{H}, \mathrm{d}, J=2.0 \mathrm{~Hz}, \mathrm{H}-6 / 6$ '), $2.76(1 \mathrm{H}, \mathrm{dd}, J=12.0,8.0 \mathrm{~Hz}, \mathrm{H}-7 \mathrm{a}), 2.92(1 \mathrm{H}, \mathrm{dd}, J=16.0,8.0 \mathrm{~Hz}, \mathrm{H}-7 \mathrm{~b}), 2.65(1 \mathrm{H}, \mathrm{m}, \mathrm{H}-8), 6.50(1 \mathrm{H}$, br s, H-2'), 2.55 (1H, m, H-8'), $3.94\left(1 \mathrm{H}, \mathrm{dd}, J=8.0,4.0 \mathrm{~Hz}, \mathrm{H}^{\prime} 9^{\prime} \mathrm{a}\right), 4.25\left(1 \mathrm{H}, \mathrm{dd}, J=8.0,4.0 \mathrm{~Hz}, \mathrm{H}-9{ }^{\prime} \mathrm{b}\right)$, 
$6.98\left(1 \mathrm{H}, \mathrm{d}, J=2.0 \mathrm{~Hz}, \mathrm{H}-2^{\prime \prime}\right), 6.74\left(2 \mathrm{H}, \mathrm{t}, J=8.0 \mathrm{~Hz}, \mathrm{H}-55^{\prime \prime}, \mathrm{H}-5^{\prime \prime \prime}\right), 6.80\left(1 \mathrm{H}, \mathrm{dd}, J=8.0,4.0 \mathrm{~Hz}, \mathrm{H}-6^{\prime \prime}\right)$, 5.46 (1H, t, $\left.J=7.0 \mathrm{~Hz}, \mathrm{H}-7^{\prime \prime} / 7^{\prime \prime \prime}\right), 3.48$ (2H, dd, $\left.J=12.0,4.0 \mathrm{~Hz}, \mathrm{H}-8^{\prime \prime} / 8^{\prime \prime \prime}\right), 7.00$ (1H, d, $\left.J=2.0 \mathrm{~Hz}, \mathrm{H}-22^{\prime \prime}\right)$, $6.82(1 \mathrm{H}, \mathrm{dd}, J=8.0,4.0 \mathrm{~Hz}, \mathrm{H}-6 " ') .{ }^{13} \mathrm{C}$ NMR $\left(400 \mathrm{MHz}, \mathrm{CD}_{3} \mathrm{OD}\right): \delta_{\mathrm{C}} 130.2(\mathrm{C}-1), 113.8(\mathrm{C}-2), 147.5(\mathrm{C}-$ 3), 145.3 (C-4), 132.7 (C-5), 114.8 (C-6), 35.8 (C-7), 47.8 (C-8), 181.6 (C-9), 130.4 (C-1'), 116.0 (C-2'), 147.5 (C-3'), 145.3 (C-4'), 133.3 (C-5'), 116.1 (C-6'), 39.3 (C-7'), 42.6 (C-8'), 73.0 (C-9'), 134.3 (C-1"), $110.6\left(\mathrm{C}-2^{\prime \prime}\right), 148.0\left(\mathrm{C}-3^{\prime \prime}\right), 148.1\left(\mathrm{C}-4^{\prime \prime}\right), 118.3\left(\mathrm{C}-5^{\prime \prime}\right), 120.0$ (C-6"), 89.1 (C-7"), 55.0 (C-8"), 64.6 (C-9"), 134.3 (C-1"'), 110.5 (C-2'"'), 149.1 (C-3"'), 134.5 (C-4"'), 118.9 (C-5"'), 119.9 (C-6"'), 89.2 (C-7"'), 55.0 $\left(\mathrm{C}-8^{\prime \prime \prime}\right), 64.6\left(\mathrm{C}-9^{\prime \prime \prime}\right), 56.4,56.4,56.6,56.7\left(\mathrm{OCH}_{3}\right)$.

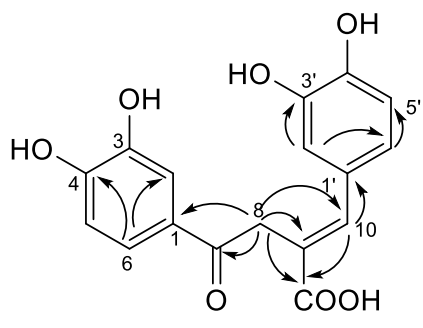

1<smiles>COc1ccc(C[C@H]2COC(=O)[C@H]2Cc2ccc(OC)c(OC)c2)cc1</smiles>

2<smiles>COc1cc([C@H]2Oc3c(OC)cc(C[C@H]4C(=O)OC[C@@H]4Cc4cc(OC)c5c(c4)[C@@H](c4ccc(O)c(OC)c4)O[C@@H]5CO)cc3[C@H]2CO)ccc1O</smiles>

Figure 1. Key HMBC correlations of compound 1 and the structures of compounds 1-3

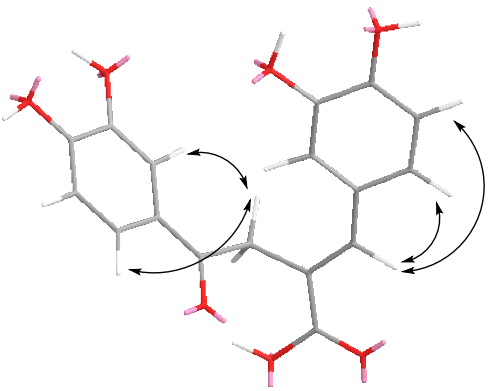

Figure 2. Key ROESY correlations (arrows) for compound 1

\section{Results and Discussion}

Compound 1, yellow oil, showed a molecular formula of $\mathrm{C}_{17} \mathrm{H}_{14} \mathrm{O}_{7}$, as deduced from HRESI (-) MS at $m / z 329.0669$ ([M-H]', calcd 329.0667). Its ${ }^{1} \mathrm{H}$ NMR spectra (Table 1) showed feature signals for one methylene at $\delta_{\mathrm{H}} 4.10(\mathrm{br} \mathrm{s}, 2 \mathrm{H})$, six aromatic proton signals at $\delta_{\mathrm{H}} 6.58(\mathrm{~d}, J=8.2,1 \mathrm{H}), 6.63(\mathrm{~d}, J=8.2,1 \mathrm{H})$, $6.70(\mathrm{br} \mathrm{s}, 1 \mathrm{H}), 6.75(\mathrm{~d}, J=8.2,1 \mathrm{H}), 7.38(\mathrm{br} \mathrm{s}, 1 \mathrm{H})$ and $7.41(\mathrm{~d}, J=8.4,1 \mathrm{H})$ assigned to two trisubstituted benzene rings, and one olefinic proton at $\delta_{\mathrm{H}} 7.74(\mathrm{~s}, 1 \mathrm{H})$. Preliminary investigation of ${ }^{13} \mathrm{C}-\mathrm{NMR}$ and DEPT spectra (Table 1) revealed a total of 17 carbon signals, consisting of nine quaternary carbons (including one olefenic, six aromatics, one ketone carbonyl and a carboxyl), seven methines (including one olefenic and six aromatics) and one methylene, indicating that compound $\mathbf{1}$ is a lignan. The NMR data is very closely related to $\beta$-(3,4-dimethoxybenzoyl)- $\alpha$-(3,4-dimethoxybenzylidene) propionic acid [14] except for missing methoxys in 1 . This change indicated that the methoxys in $\beta$-(3,4-dimethoxybenzoyl)- $\alpha$-( $3,4-$ dimethoxybenzylidene) propionic acid was reduced to the hydroxyls in $\mathbf{1}$, which can be observed in ${ }^{13} \mathrm{C}$ NMR and DEPT spectrum. The key HMBC correlations from H-8 to C-1, C-7, C-9, C-10 and C-11, and from $\mathrm{H}-10$ to $\mathrm{C}-1$ ' confirmed the connection between the straight chain fragment and the two benzene rings (Figure 1). The cis-trans isomerism of double bond at $\Delta^{9(10)}$ was trans can be confirmed by the cross-peaks 
of $\mathrm{H}-8 / \mathrm{H}-2 / \mathrm{H}-6$ and $\mathrm{H}-10 / \mathrm{H}-5^{\prime} / \mathrm{H}-6^{\prime}$ in ROESY spectrum (Figure 2). Thus, 1 was elucidated to be amaurolignan A.

These skeleton type of pericarbonyl lignans were rare in nature, most of them are intermediates in organic synthesis reactions, like the Perkin condensation product $\alpha$-arylidine- $\beta$-benzoyl propionic acid [1518]. To be best of our knowledge, this is the first report of natural compound with this skeleton from the fungus and the first time from Ganodermataceae.

Table 1. ${ }^{1} \mathrm{H}$ NMR and ${ }^{13} \mathrm{C}$ NMR data for compound 1 (at $400 \mathrm{MHz}$ in $\mathrm{CD}_{3} \mathrm{OD}, \delta$ in ppm, $J$ in $\mathrm{Hz}$ )

\begin{tabular}{ccc}
\hline Position & H & $\mathbf{C}$ \\
\hline 1 & & 128.9 \\
2 & $7.38(1 \mathrm{H}, b r s)$ & 114.7 \\
3 & & 150.9 \\
4 & $6.75(1 \mathrm{H}, d, J=8.2)$ & 145.0 \\
5 & $7.41(1 \mathrm{H}, d, J=8.2)$ & 114.5 \\
6 & $4.10(2 \mathrm{H}, b r s)$ & 121.9 \\
7 & & 197.3 \\
8 & $7.74(1 \mathrm{H}, s)$ & 37.5 \\
9 & & 124.5 \\
10 & & 142.1 \\
11 & $6.63(1 \mathrm{H}, d, J=8.2)$ & 170.2 \\
$1^{\prime}$ & & 127.0 \\
$2^{\prime}$ & & 115.0 \\
$3^{\prime}$ & $6.58(1 \mathrm{H}, d d, J=8.2,1.5)$ & 144.9 \\
$4^{\prime}$ & $6.70(1 \mathrm{H}, d, J=1.5)$ & 146.4 \\
$5^{\prime}$ & & 121.5 \\
$6^{\prime}$ & & 115.9 \\
\hline
\end{tabular}

\section{Acknowledgments} 31560099).

Financial support of the National Natural Science Foundation of China (No. 31600282 and

\section{Supporting Information}

Supporting information accompanies this paper on http://www.acgpubs.org/journal/records-ofnatural-products

\section{ORCID}

Miao Dong: 0000-0002-0003-9402

Zuhong Ma: 0000-0002-1368-4902

Qiaofen Yang: 0000-0003-3462-5079

Qiuyue Hu: 0000-0002-9236-7963

Yanqing Ye: 0000-0003-4244-5385

Min Zhou: 0000-0003-1896-9832

\section{References}

[1] Y. Cao, S. H. Wu and Y. C. Dai (2012). Species clarification of the prize medicinal Ganoderma mushroom "Lingzhi”, Fungal Divers. 56, 49-62.

[2] A. H. Aly, A. Debbab and P. Proksch (2011). Fifty years of drug discovery from fungi, Fungal Divers. 50, 319.

[3] Y. C. Dai, Z. L. Yang, B. K. Cui, C. J. Yu and L. W. Zhou (2009). Species diversity and utilization of medicinal 
mushrooms and fungi in China (Review), Int. J. Med. Mushrooms. 11 (3), 287-302.

[4] D. D. De Silva, S. Rapior, F. Fons, A. H. Bahkali and K. D. Hyde (2012). Medicinal mushrooms in supportive cancer therapies: an approach to anti-cancer effects and putative mechanisms of action, Fungal Divers. 55, 135 .

[5] Y. H. Gao and S. F. Zhou (2003). Cancer prevention and treatment by Ganoderma, a mushroom with medicinal properties, Food Rev. Int. 19 (3), 275-325.

[6] Z. B. Lin (2009). Lingzhi: from mystery to science. Peking University Medical Press, Beijing.

[7] B. G. Jung, J. A. Lee and B. J. Lee (2012). Immunoprophylactic effects of shiitake mushroom (lentinula edodes) against bordetella bronchiseptica in mice, J. Microbiol. 50 (6), 1003-1008.

[8] C. J. Chen, R. Vijaya Krishna, C. C. Tsai, W. H. Wu, L. K. Chao, K. H. Hwang, C. M. Chien, H. Y. Chang and S. T. Chen (2010). Structure and functions of gamma-dodecalactone isolated from Antrodia camphorata for NK cell activation, Bioorgan. Med. Chem. 18 (18), 6896-6904.

[9] C. Jiao, Y. Z. Xie, X. Yang, H. Li, X. M. Li, H. H. Pan, M. H. Cai, H. M. Zhong and B. B. Yang (2013). Anticancer activity of amauroderma rude, Plos One 8 (6), e66504.

[10] P. M. Chan, G. Kanagasabapathy, Y. S. Tan, V. Sabaratnam and U. R. Kuppusamy (2013). Amauroderma rugosum (blume \& t. nees) torrend: nutritional composition and antioxidant and potential anti-inflammatory properties, Evid-Based. Compl. Alt. 2013 (4), 304713.

[11] S. S. Zhang, Q. Y. Ma, X. S. Zou, H. F. Dai, S. Z. Huang, Y. Luo, Z. F. Yu, H. R. Luo and Y. X. Zhao (2013). Chemical constituents from the fungus Amauroderma amoiensis and their in vitro acetylcholinesterase inhibitory activities, Planta Med. 79, 87-91.

[12] R. Tundis, G. Statti, F. Menichini and M. F. Delle (2000). Arctiin and onopordopicrin from carduus micropterus ssp. Perspinosus, Fitoterapia 71 (5), 600-601.

[13] K. Umehara, M. Nakamura, T. Miyase, M. Kuroyanagi and A. Ueno (1996). Studies on differentiation inducers. vi. lignan derivatives from arctium fructus. (2), Chem. Pharm. Bull. 44 (12), 2300-2304.

[14] M. Rischmann, R. Mues, H. Geiger, H. J. Laas and T. Eicher (1989). Isolation and synthesis of 6,7-dihydroxy4-(3,4-dihydroxyphenyl)naphthalene-2-carboxylic acid from pellia epiphylla, Phytochemistry 28 (3), 867-869.

[15] T. Chaudhari, S. Deo and F. Inam (2013). Natural \& Synthetic 1-Phenylnaphthalene Lignans. Natural \& synthetic 1-Phenylnaphthalene lignans: Immunomodulation. LAP LAMBERT Academic Publishing.

[16] R. D. Haworth and D. Woodcock (1938). 154. the constituents of natural phenolic resins. part xi. synthesis of 2- and 3-methyl-6 : 7-dimethoxy-1-veratrylnaphthalenes, J. Chem. Soc. 54, 809-813.

[17] Z. Ali, S. Deo and F. Inam (2013). A recyclable and highly effective sulfamic acid/etoh catalytic system in synthesis and natural product chemistry of lignan compounds, Asian J. Chem. 25 (2), 899-904.

[18] S. Deo, T. Chaudhari and F. Inam (2015). Cheminform abstract: microwave assisted perkin reaction for the synthesis of $\alpha$-arylidine- $\gamma$-phenyl- $\Delta, \beta, \gamma$-butenolides, Cheminform 45 (37), 111-117.

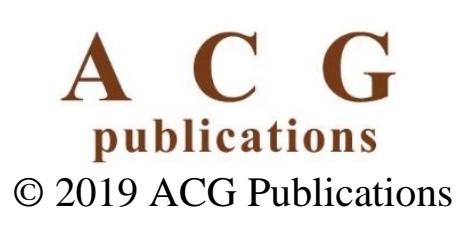

